\title{
Magnetic white dwarfs in the Early Data Release of the Sloan Digital Sky Survey
}

\author{
B. T. Gänsicke ${ }^{1}$, F. Euchner ${ }^{2}$, and S. Jordan ${ }^{3}$ \\ 1 Department of Physics and Astronomy, University of Southampton, Southampton SO17 1BJ, UK \\ 2 Universitäts-Sternwarte, Geismarlandstr. 11, 37083 Göttingen, Germany \\ 3 Institut für Astronomie und Astrophysik, Universität Tübingen, Sand 1, 72076 Tübingen, Germany
}

Received 26 July 2002 / Accepted 23 August 2002

\begin{abstract}
We have identified 7 new magnetic DA white dwarfs in the Early Data Release of the Sloan Digital Sky Survey. Our selection strategy has also recovered all the previously known magnetic white dwarfs contained in the SDSS EDR, KUV 03292+0035 and HE0330-0002. Analysing the SDSS fibre spectroscopy of the magnetic DA white dwarfs with our state-of-the-art model spectra, we find dipole field strengths $1.5 \mathrm{MG} \leq B_{\mathrm{d}} \leq 63 \mathrm{MG}$ and effective temperatures $8500 \leq T_{\text {eff }} \leq$ $39000 \mathrm{~K}$. As a conservative estimate, we expect that the complete SDSS will increase the number of known magnetic white dwarfs by a factor 3 .
\end{abstract}

Key words. stars: white dwarfs - stars: magnetic fields - stars: atmospheres

\section{Introduction}

The population of magnetic white dwarfs spans an enormous parameter space in magnetic field strength $B$, effective temperature $T_{\text {eff }}$, rotational period $P_{\text {rot }}$, atmospheric abundances, and mass $M_{\mathrm{wd}}$ - with the number of accurate measurements per parameter dimension decreasing in this sequence. Despite intense spectroscopic and polarimetric surveys carried out over the last two decades (e.g. Schmidt \& Smith 1995; Putney 1995; Putney 1997; Hagen et al. 1987; Reimers et al. 1994; Reimers et al. 1996; Reimers et al. 1998), only $\sim 65$ magnetic white dwarfs are known at present (Jordan 2001; Wickramasinghe \& Ferrario 2000). The small size of this sample seriously hampers the progress of our understanding of the origin of the strong magnetic fields found in a small fraction (few \%) of all white dwarfs, as well as of the evolution of these exotic stars.

The Sloan Digital Sky Survey (SDSS), the largest spectroscopic survey carried out to date, samples a great variety of galactic and extragalactic objects at high galactic latitudes. Due to the partial overlap in colour space between white dwarfs and quasars, it can be expected that the SDSS will result in the identification of a large number of white dwarfs and, hence, in a significantly increased sample of known magnetic white dwarfs. Here we describe the sample of magnetic white dwarfs identified in the Early Data Release of the SDSS.

Send offprint requests to: $\mathrm{B}$. Gänsicke,

e-mail: btg@astro.soton.ac.uk

\section{Sloan Digital Sky Survey observations}

The Sloan Digital Sky Survey is an ambitious project which will provide deep CCD imaging of $\sim 10000 \mathrm{deg}^{2}$ of the north Galactic cap in five optical bands, as well as lowresolution spectroscopy for $\sim 10^{6}$ astronomical objects selected on the base of their colours derived from the imaging data. Both, imaging and spectroscopy are carried out with the purpose-built SDSS $2.5 \mathrm{~m}$ telescope located at Apache Point Observatory. The SDSS follow-up spectroscopy is obtained using two fibre-fed spectrographs that allow to observe more than 600 objects simultaneously. The spectrographs cover 3800$9200 \AA$ at a spectral resolution of $R \simeq 1800$. The reduction of the spectral data is performed by an automated software pipeline. For a technical description of the survey, see York et al. (2000). Even though the main purpose of this project is to produce the most comprehensive galaxy and quasar redshift survey to date (the majority of the spectrograph fibres are allocated to galaxy/quasar candidates) it delivers a vast amount of data for detailed studies of the galactic stellar population. A few recent examples of such studies are the discoveries of a very cool white dwarf (Harris et al. 2001), of a sample of cataclysmic variables (Szkody et al. 2002), and of a sample of carbon stars (Margon et al. 2002).

The Early Data Release (EDR) of the SDSS (Stoughton et al. 2002) contains imaging data for $462 \mathrm{deg}^{2}$ and a total of 54008 fibre spectra obtained in that region. 
Table 1. Confirmed and candidate magnetic white dwarfs from the SDSS EDR. The equinox/epoch 2000 coordinates are coded in the object designation. The objects are classified in the SDSS EDR as stellar (S) or unknown (U). The spectra are uniquely identified in the SDSS database by the combination of the Plate ID, the modified Julian date, and the fibre ID of the observation. The magnitudes listed here are from the associated imaging data.

\begin{tabular}{|c|c|c|c|c|c|c|c|c|c|c|}
\hline MWD & Class. & Sample & Spectrum ID & Obs. date & Exp. & $u^{*}$ & $g^{*}$ & $\overline{r^{*}}$ & 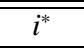 & $\overline{z^{*}}$ \\
\hline SDSS J030407.40-002541.7 & $\mathrm{DA}$ & $S$ & $411-51817-172$ & 2000-09-30 10:37 & $2700 \mathrm{~s}$ & 18.06 & 17.77 & 17.95 & 18.09 & 18.37 \\
\hline SDSS J033145.69+004517.0* & DA & $S$ & $415-51810-370$ & 2000-09-23 08:34 & $3600 \mathrm{~s}$ & 17.31 & 17.23 & 17.49 & 17.74 & 18.00 \\
\hline SDSS J033320.37+000720.7 ${ }^{+}$ & DB? & $\mathrm{U}$ & $415-51810-492$ & $2000-09-2308: 34$ & $3600 \mathrm{~s}$ & 17.02 & 16.52 & 16.41 & 16.34 & 16.48 \\
\hline SDSS J034511.11+003444.3 & DA & $S$ & $416-51811-590$ & 2009-09-24 09:35 & $3600 s$ & 19.11 & 18.63 & 18.52 & 18.49 & 18.50 \\
\hline SDSS J121635.37-002656.2 & DA & $\mathrm{U}$ & $288-52000-276$ & 2001-04-01 06:24 & $3602 \mathrm{~s}$ & 19.85 & 19.57 & 19.83 & 20.05 & 20.11 \\
\hline SDSS J122209.44+001534.0 & DA & $\mathrm{U}$ & 289-51990-349 & 2001-03-22 06:25 & $3604 \mathrm{~s}$ & 20.51 & 20.23 & 20.47 & 20.65 & 21.04 \\
\hline SDSS J172045.37+561214.9 & DA & $\mathrm{U}$ & $367-51997-461$ & 2001-03-22 11:51 & $6302 \mathrm{~s}$ & 20.00 & 20.11 & 20.49 & 20.76 & 21.29 \\
\hline SDSS J172329.14+540755.8 & DA & $S$ & $359-51821-415$ & $2000-10-03$ 03:59 & $4500 \mathrm{~s}$ & 19.14 & 18.81 & 18.90 & 19.04 & 19.30 \\
\hline SDSS J232248.22+003900.9 & DA & $S$ & $383-51818-421$ & 2000-09-07 08:10 & $3600 s$ & 18.91 & 19.02 & 19.31 & 19.62 & 19.82 \\
\hline SDSS J232337.55-004628.2 & DA? & $S$ & $383-51818-215$ & 2000-10-01 04:29 & $3600 s$ & 17.88 & 18.00 & 18.27 & 18.52 & 18.78 \\
\hline
\end{tabular}

$*$ KUV $03292+0035+=$ HE 0330-0002.

\section{Selection strategy}

We have used the SDSS Science Archive Query Tool (sdssQT) to select all entries from the EDR spectroscopic data base that were classified as "star" by the processing pipeline ( ppecClass $=1$ or 6 ). A total of 7072 spectra were downloaded $^{1}$ as FITS files. All spectra were visually inspected for peculiar absorption structures, especially for broad absorption troughs near $\mathrm{H} \alpha$ and $\mathrm{H} \beta$ as possible indicators for Zeeman splitting in a magnetic field of a few to a few tens MG. Discarding a number of spectra with very poor signalto-noise ratio, six objects were selected (those marked " $\mathrm{S}$ " in Table 1). The details of the observations are listed in Table 1. A cross-check with the Simbad database revealed that SDSS J033145.69+004517.0 is a known magnetic DA white dwarf, discovered by Wegner et al. (1987) during follow-up observations of the Kiso survey for UV-excess objects.

Figure 1 shows colour-colour diagrams for all 7072 "stellar" objects from the EDR spectroscopic database. It is apparent that all six magnetic white dwarfs candidates fall well within the "hot star" quadrant of the $u^{*}-g^{*}$ vs. $g^{*}-r^{*}$ diagram - the vast majority of objects in this colour space are white dwarfs and subdwarfs. Based on the colour space confinement of the objects selected among the "stellar" EDR fraction, we extended our search for magnetic white dwarfs to the spectra classified as "non-stellar" by the SDSS processing pipeline ( $\operatorname{specClass}=0,2,3$ or 4 ). We downloaded and visually inspected 3224 spectra whose associated imaging data satisfies $u^{*}-g^{*}<0.6$ and $g^{*}-i^{*}<0.5$, and selected four additional magnetic white dwarf candidates (Table 1), all of which were classified as "unknown" (specClass $=0)$. Cross correlation with Simbad showed that SDSS J033320.37+000720.7 is a known magnetic white dwarf discovered in the Hamburg/ESO Survey, probably with a helium-rich atmosphere (Reimers et al. 1998; Schmidt et al. 2001).

The SDSS fibre spectra of the objects listed in Table 1 are displayed in Figs. 2 to 4 . We have checked the $u^{*} g^{*} r^{*} i^{*} z^{*}$

\footnotetext{
${ }^{1}$ http://sdssdp7. fnal.gov/cgi-bin/das/spectra
}

magnitudes derived from the EDR spectrum and found the spectroscopic magnitudes to be $\lesssim 0.2$ mag fainter than the photometric magnitudes for all but the faintest objects, which is in good agreement with the accuracy of the absolute flux calibration of the SDSS spectroscopy quoted by Stoughton et al. (2002). Monochromatic fluxes at the effective wavelengths of the $g^{*}, r^{*}$, and $i^{*}$ filters derived from the associated SDSS imaging data are plotted along with the fibre spectra.

\section{Analysis}

We have used the code of Euchner et al. (2002) to confirm the magnetic white dwarf nature of the candidates selected from the SDSS EDR, and to derive their fundamental properties. In brief, this code computes flux and circular polarisation spectra for a magnetic white dwarf with an almost arbitrary field topology by adding up appropriately weighted model spectra for a large number of surface elements. To save time, the code makes use of a library of several 10000 synthetic spectra calculated with the stellar atmosphere program developed by Jordan between 1988 and 2002, covering a wide range in magnetic field strength $B$, effective temperature $T_{\text {eff }}$ and angle between the line of sight and the direction of the magnetic field at the stellar surface, $\psi$. Limb darkening is accounted for by a simple linear scaling law. The magnetic field configuration of the white dwarf is described by a multipole expansion of the scalar magnetic potential. The individual multipole components may be independently oriented with respect to the rotation axis of the white dwarf and offset with respect to its centre, allowing for rather complex surface field topologies. Additional parameters are the white dwarf effective temperature and the inclination of either the rotation or the magnetic axis with respect to the line of sight. Observed spectra can be fitted using an evolutionary strategy with a least-squares quality function.

For the analysis of the SDSS fibre spectra presented here, we assumed the simplest field topology, a centred dipole, resulting in four free parameters: the magnetic dipole field strength $B_{\mathrm{d}}$, the effective temperature $T_{\text {eff }}$, the inclination of 

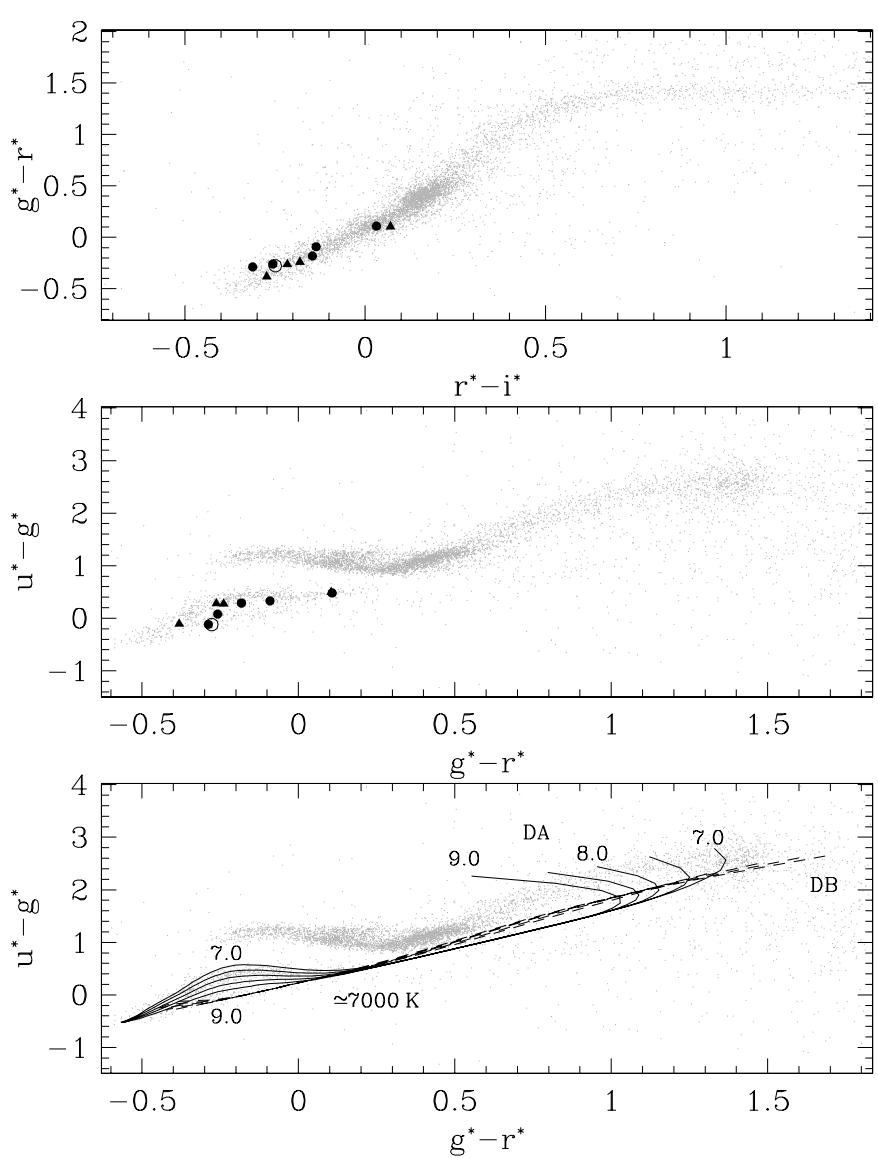

Fig. 1. SDSS colour-colour diagrams of the stellar component of the EDR. The magnetic white dwarf candidates selected from the "stellar" and "non-stellar" fraction of the EDR sample are plotted as filled circles and filled triangles, respectively (top and middle panel). Hot stars - mainly white dwarfs and subdwarfs - are clearly separated from the bulk of the main sequence stars in the lower left quadrant of the $u^{*}-g^{*} / g^{*}-r^{*}$ diagram. The bottom panel shows DA (solid lines) and DB (dashed lines) cooling tracks of non-magnetic white dwarfs for surface gravities $\log g=7.0$ to 9.0, computed from model spectra (P. Bergeron, private communication). The DA cooling tracks range from $T_{\text {eff }}=100000 \mathrm{~K}$ (left) to $1500 \mathrm{~K}$ (right) and cross each other in the gap between the hot stars and the main sequence at $T_{\text {eff }} \simeq 7000 \mathrm{~K}$. The DB cooling tracks range from $T_{\text {eff }}=30000 \mathrm{~K}$ (left) to $4000 \mathrm{~K}$ (right). The magnetic white dwarf candidates discussed in this paper are well confined within the hot star population.

the magnetic axis against the line of sight, $i$, and the flux normalisation.

We were not able to obtain in all cases a fit that consistently satisfies both the continuum flux distribution and the strength of the Zeeman absorption features. A similar discrepancy between the strength of the Balmer lines and the optical continuum slope has been reported by Achilleos et al. (1991) for the low-field white dwarfs WD 0159-032 $\left(B_{\mathrm{d}}=6 \mathrm{MG}, T_{\text {eff }}=26000 \mathrm{~K}\right)$ and, to a lesser degree, for WD 0307-428 $\left(B_{\mathrm{d}}=10 \mathrm{MG}, T_{\mathrm{eff}}=\right.$ $25000 \mathrm{~K})$.

For non-magnetic white dwarfs, the effective temperature can be derived very reliably from fitting the Balmer line profiles (e.g. Bergeron et al. 1992; Finley et al. 1997) ${ }^{2}$. However, in the case of magnetic white dwarfs, is is presently not yet possible to compute detailed line profiles because there exists no theory that consistently describes Stark broadening in the presence of substantial magnetic fields (see, e.g., Jordan 1992), and, as a result, the computed equivalent widths of the individual Zeeman components are subject to systematic uncertainties. Consequently, effective temperatures which are derived from fitting the Balmer lines alone are less reliable in the case of magnetic white dwarfs than in the case of non-magnetic white dwarfs, and may be in apparent disagreement with temperature estimates derived from the continuum slope.

We provide below effective temperature estimates derived from (a) fitting only the Balmer lines (normalising the continuum slope of the model spectrum to that of the observed spectrum), and (b) fitting the continuum slope.

SDSS J030407.40+002541.7: The EDR spectrum of SDSS 0304 contains the H $\alpha$ Zeeman triplet split by $\sim \pm 200 \AA$, as well as numerous Zeeman components of $\mathrm{H} \beta$ and $\mathrm{H} \gamma$ (Fig.2). The data are well-fit with a $T_{\text {eff }}=15000 \mathrm{~K}$ white dwarf with a dipole field strength $B_{\mathrm{d}}=10.8 \mathrm{MG}$ and a magnetic inclination $i=50^{\circ}$ (where $i=90^{\circ}$ corresponds to an equator-on view). The model spectrum provides a good match for both the continuum slope and the strength of the Zeeman absorption lines. The most noticeable shortcoming of this fit is the poor agreement in the $\sigma^{-}$component of $\mathrm{H} \beta$. The lack of a consistent description of the combined Zeeman and Stark effects in this magnetic field range prevents a perfect fit to the line profiles.

SDSS J033145.69+004517.0 = KUV 03292+0035: The spectrum of SDSS 0331 is remarkably similar to that of SDSS 0304, and we find from a fit to the Balmer lines $T_{\text {eff }}=26500 \mathrm{~K}, B=12.1 \mathrm{MG}$, and $i=55^{\circ}$. A fit to the continuum slope suggests a similar temperature, $T_{\text {eff }} \sim 25-30000 \mathrm{~K}$. Jordan (1993) derived for KUV 03292+0035 $B_{\mathrm{d}}=12 \mathrm{MG}$, $T_{\text {eff }}=19000 \mathrm{~K}$, and a magnetic inclination of $i=20^{\circ}$, which is in general agreement with the Balmer line analysis presented here. The difference in $i$ between the two analyses might be suggestive of a rotational variation of the Zeeman pattern, but the presently available data are not sufficient to convincingly confirm this possibility.

Similar to SDSS 0304, our fit to the Zeeman absorption structures fails to reproduce the strength of the $\mathrm{H} \beta \sigma^{-}$component. Close inspection of the EDR spectrum of SDSS 0331 reveals in addition sharp $\sigma^{+/-}$components of $\mathrm{H} \alpha$ at $6435 \AA$ and $6691 \AA$, respectively (see inset in Fig. 2). These narrow features cannot be reproduced by our simple dipole model, suggesting a more complex field topology. Their position corresponds to a field of $\sim 5.5 \mathrm{MG}$, substantially lower than the dipole field strength derived from our fit. A more detailed investigation of the field topology has to await additional data and would be especially promising if a rotational variation of the Zeeman features will be detected by future measurements.

${ }^{2}$ Generally, in both magnetic and non-magnetic white dwarfs with $T_{\text {eff }}$ exceeding $20000 \mathrm{~K}$ the slope of the continuum is becoming insensitive to the temperature as it gradually approaches a RayleighJeans distribution. 

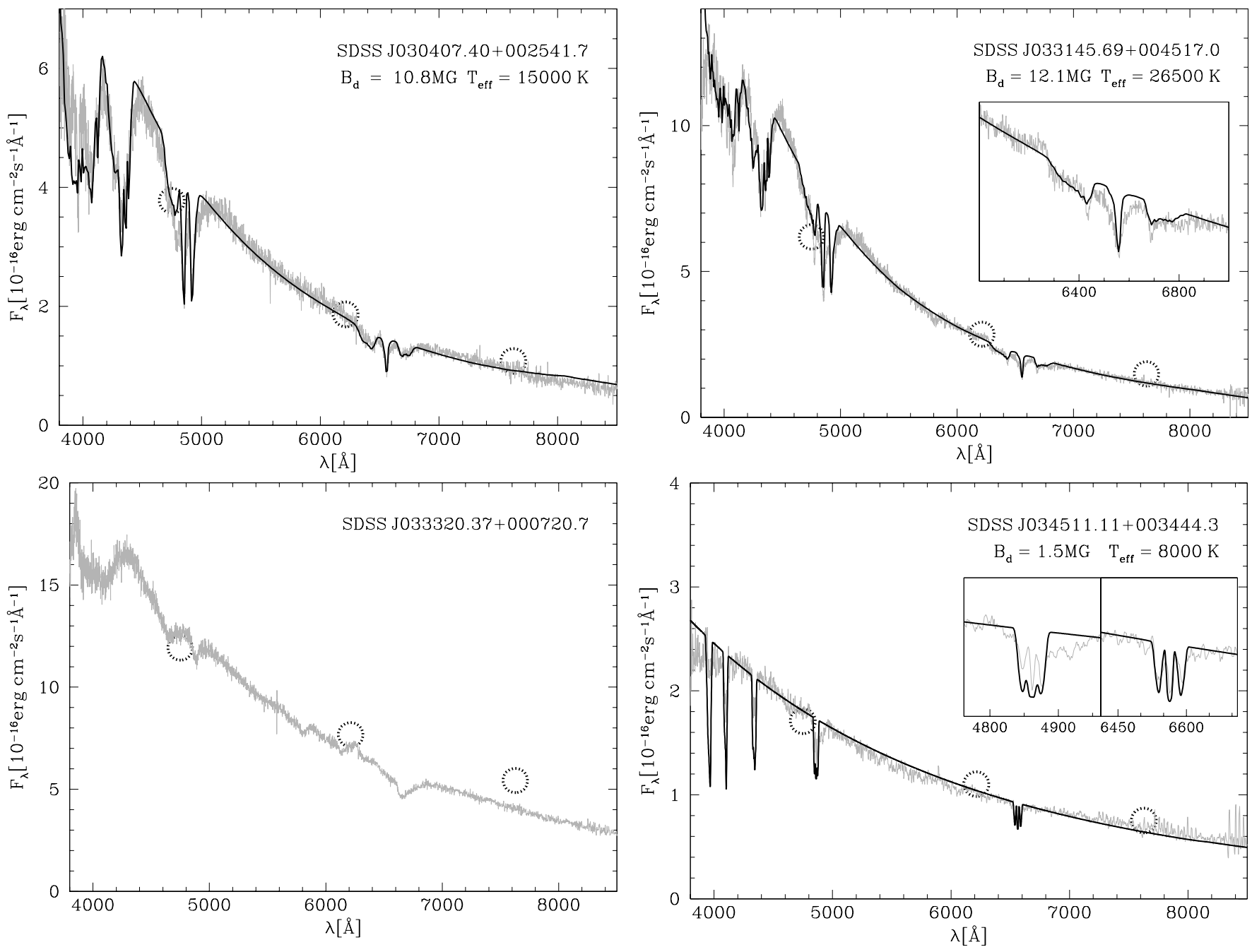

Fig. 2. Magnetic white dwarfs from the SDSS EDR. Gray line: SDSS fibre spectroscopy. Black line: best-fit magnetic white dwarf model spectrum assuming a simple dipole field configuration. Open circles: monochromatic $g^{*}, r^{*}$, and $i^{*}$ fluxes at the effective wavelengths (Fukugita et al. 1996) calculated from the magnitudes of the stars in the associated imaging data (Table 1).

SDSS J033320.37+000720.7 = HE 0330-0002:

SDSS 0333 is one of the four magnetic white dwarfs that we have selected among the objects classified as "unknown" by the SDSS processing pipeline. It has been identified before as a peculiar white dwarf in the Hamburg/ESO survey for bright quasars (HE 0330-0002, Reimers et al. 1998). Reimers et al. (1998) noted that the absorption features observed in HE 0330-0002 are not readily explained with either hydrogen or helium transitions. Schmidt et al. (2001) confirmed HE 0330-0002 as a magnetic white dwarf by the detection of circular polarisation and suggested a helium-dominated atmosphere for this star. Considering the previous analyses of this object, we did not attempt to fit the observed spectrum with our pure hydrogen atmosphere models.

It is interesting to note that HE 0330-0002 appeared to be a rather cool white dwarf in the observations of both Reimers et al. (1998) and Schmidt et al. (2001). The latter authors suggested $T_{\text {eff }} \sim 6000-7000 \mathrm{~K}$ based on the energy distribution of the continuum. While the magnitudes derived from the SDSS imaging data are consistent with such a low temperature, the SDSS fibre spectrum displays a significantly bluer slope, corresponding to $T_{\text {eff }} \sim 12000 \mathrm{~K}$. Considering that the SDSS imaging data was obtained on 1998 September 25, whereas the SDSS spectroscopy was obtained on 2000 September 23, the discrepancy in flux may suggest intrinsic variability. We also note that the USNO-A2.0 catalogue lists HE0330-0002 with $B=16.2$, whereas Reimers et al. (1998) give $B_{\mathrm{J}}=16.8$. Schmidt et al. (2001) observed HE0330-0002 twice over a few days and reported no noticeable change on that time scale. Photometric and/or spectroscopic monitoring HE 0330-0002 is encouraged to test for long-term variability.

SDSS J034511.11+003444.3: SDSS 0345 is the star with the lowest field in our sample, we find $B \simeq 1.5 \mathrm{MG}$ and $i \simeq 0^{\circ}$ from a fit to the $\mathrm{H} \alpha$ and $\mathrm{H} \beta$ Zeeman components. It is also the coldest star in our sample, the strength of the Balmer lines suggests $T_{\text {eff }} \simeq 8000 \mathrm{~K}$, whereas a fit to the continuum results in $T_{\text {eff }} \simeq 9000 \mathrm{~K}$.

SDSS J121635.37-002656.2: SDSS 1216 has been selected as a magnetic white dwarf candidate among the objects classified as "unknown" in the SDSS EDR database because of the complex absorption structure near $\mathrm{H} \beta$ and $\mathrm{H} \gamma$ seen in its spectrum. A dipole fit to the Balmer lines confirms the 

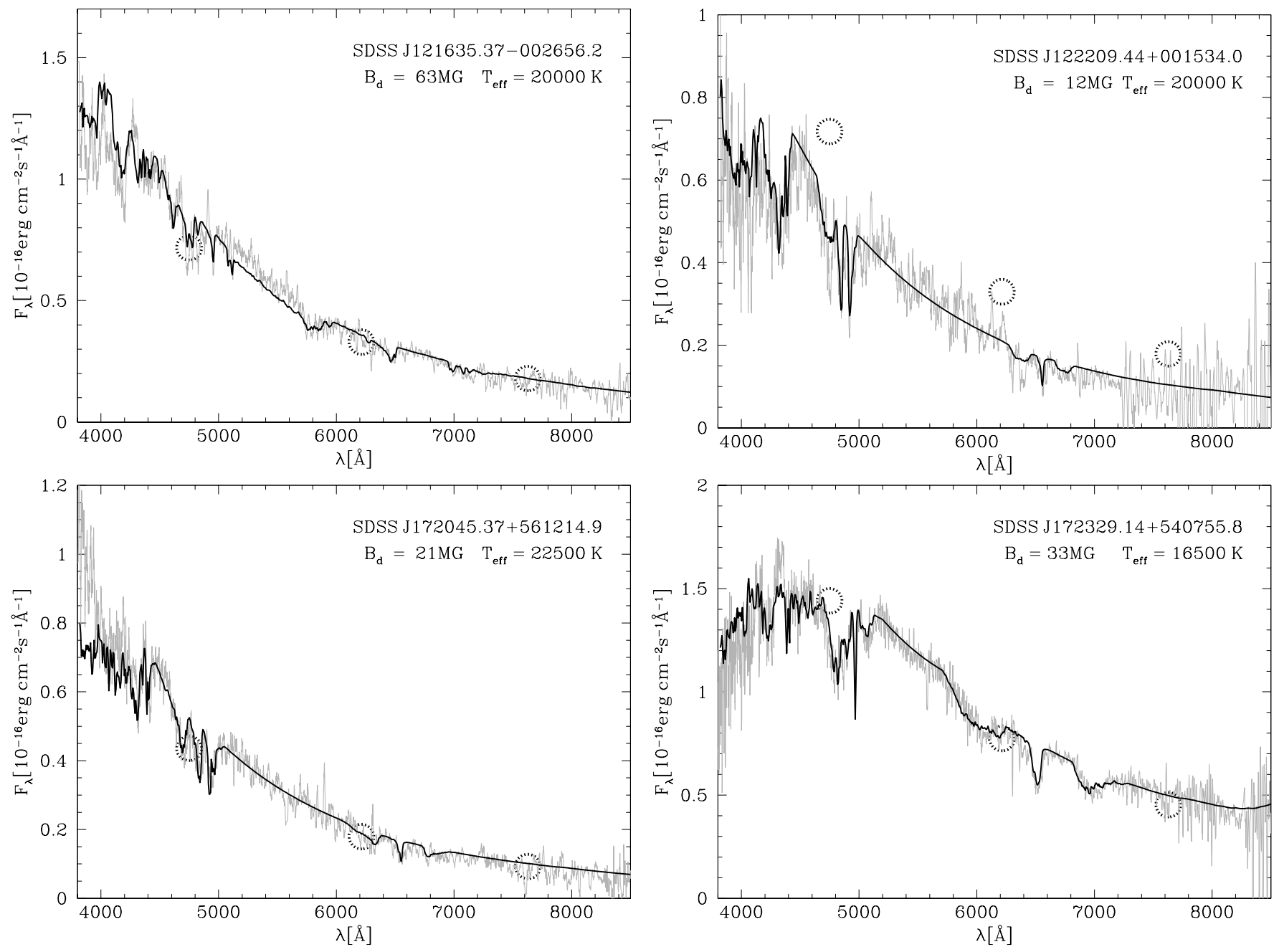

Fig. 3. Magnetic white dwarfs from the SDSS EDR (continued), see Fig. 2 for further details.

object as a magnetic white dwarf with $B_{\mathrm{d}}=63 \mathrm{MG}$, the highest field in the sample of magnetic white dwarfs presented here, $T_{\text {eff }}=20000 \mathrm{~K}$, and $i=75^{\circ}$. The effective temperature derived from the fit to the Balmer lines is somewhat higher than that derived from the continuum slope, $T_{\text {eff }}=15000 \mathrm{~K}$.

SDSS J122209.44+001534.0: SDSS 1222, classified as "unknown" in the SDSS EDR database, is the faintest object in our sample of SDSS magnetic white dwarfs and the signalto-noise ratio of the fibre spectrum is consequently very low. Nevertheless, the Zeeman triplet of $\mathrm{H} \beta$ split in a moderate field is clearly recognised, and we find $B=12 \mathrm{MG}, T_{\mathrm{eff}}=20000 \mathrm{~K}$, and $i=40^{\circ}$. The effective temperature derived from the fit to the Balmer lines is broadly consistent with the continuum slope.

SDSS J172045.37+561214.9: The spectrum of SDSS 1720, the fourth magnetic white dwarf candidate selected from the "unknown" class in the SDSS EDR database, displays a broad $\mathrm{H} \beta$ triplet. The parameters derived from a dipole fit to the Balmer lines are are $B_{\mathrm{d}}=21 \mathrm{MG}$, $T_{\text {eff }}=22500 \mathrm{~K}$, and $i=85^{\circ}$. The slope of the continuum is consistent with a temperature in the range $20-30000 \mathrm{~K}$.

SDSS J172329.14+540755.8: SDSS 1723 has been selected because of the broad $\mathrm{H} \alpha$ triplet and the complex $\mathrm{H} \beta$ absorption detected in its spectrum. The turnover of the continuum at $\lambda \lesssim 4200 \AA$ indicates a relatively low temperature. Our dipole fit to the Balmer lines results in $B_{\mathrm{d}}=33 \mathrm{MG}$, $T_{\text {eff }}=16500 \mathrm{~K}$, and $i=35^{\circ}$. Fitting the continuum slope of the spectrum of SDSS 1723 results in a significantly lower value for the effective temperature, $T_{\text {eff }} \simeq 10000 \mathrm{~K}$.

SDSS J232248.22+003900.9: The fibre spectrum of SDSS 2322 contains relatively weak but broad absorption structures near $\mathrm{H} \beta$ and $\mathrm{H} \gamma$ in a blue continuum. A dipole fit confirms the object as a magnetic white dwarf with $B_{\mathrm{d}}=$ $13 \mathrm{MG}, T_{\mathrm{eff}}=39000 \mathrm{~K}$, and $i=25^{\circ}$. The temperature derived from the continuum slope is $T_{\text {eff }} \simeq 25-30000 \mathrm{~K}$.

SDSS J232337.55-004628.2: The spectrum of SDSS 2323 resembles that of SDSS 1222 with broad absorption features of $\mathrm{H} \beta / \mathrm{H} \gamma$ and a blue continuum. However, we were not able to find a satisfying fit with our dipole models. Figure 4 shows the fibre spectrum of SDSS 2323 along with an illustrative dipole model computed for $B=30 \mathrm{MG}, T_{\text {eff }}=20000 \mathrm{~K}$, and $i=0^{\circ}$. Close inspection of the spectrum reveals that a number of the narrow absorption lines present in the spectrum could be explained with He I transitions. However, while the width of the $\mathrm{H} \beta$ and $\mathrm{H} \gamma$ absorption troughs clearly suggests the (magnetic?) white dwarf nature of this object, the 

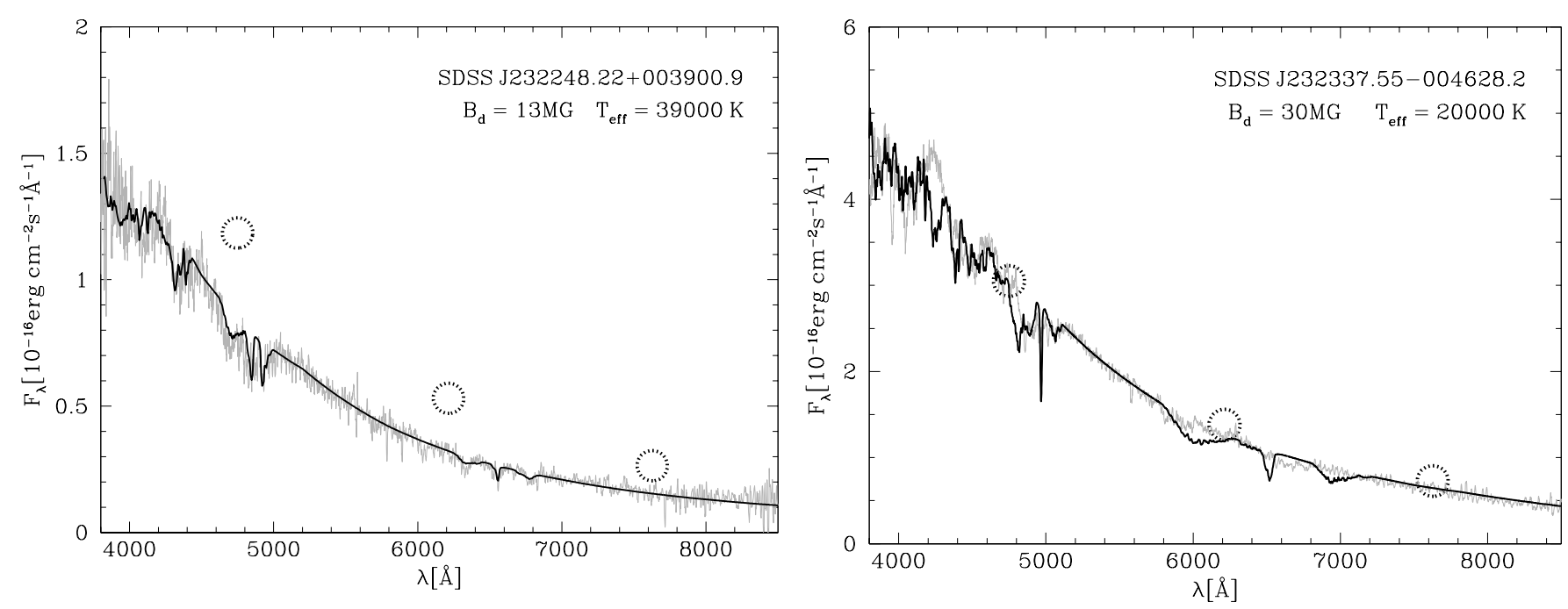

Fig. 4. Magnetic white dwarfs from the SDSS EDR (continued), see Fig. 2 for further details.

Table 2. Best-fit parameter for the confirmed DA magnetic white dwarfs. Two effective temperature estimates are given, based in fits to the Balmer lines and to the continuum slope.

\begin{tabular}{lrrr}
\hline \hline MWD & $B_{\mathrm{d}}[\mathrm{MG}]$ & $\begin{array}{r}T_{\text {eff }}[\mathrm{K}] \\
\text { lines }\end{array}$ & $\begin{array}{r}T_{\text {eff }}[\mathrm{K}] \\
\text { continuum }\end{array}$ \\
\hline SDSS J030407.40-002541.7 & 10.8 & 15000 & 15000 \\
SDSS J033145.69+004517.0 & 12.1 & 26500 & $25-30000$ \\
SDSS J034511.11+003444.3 & 1.5 & 8000 & 9000 \\
SDSS J121635.37-002656.2 & 63 & 20000 & 15000 \\
SDSS J122209.44+001534.0 & 12 & 20000 & $15-20000$ \\
SDSS J172045.37+561214.9 & 21 & 22500 & $20-30000$ \\
SDSS J172329.14+540755.8 & 33 & 16500 & 10000 \\
SDSS J232248.22+003900.9 & 13 & 39000 & $25-30000$ \\
\hline
\end{tabular}

potential He I lines are too narrow for an origin in a high gravity atmosphere. Inspection of the SDSS $r^{*}$ image did not reveal anything suspicious about this object (e.g. a close visual companion). Additional observations of SDSS 2323 are needed to clarify the nature of this object.

\section{Discussion and conclusions}

The population of known magnetic white dwarfs presently comprises $\sim 65$ stars (Wickramasinghe \& Ferrario 2000). Considering that the first magnetic white dwarf (Grw $+70^{\circ} 8047$ ) has been discovered nearly 60 years ago, the "average" discovery rate of these stars has been $\sim 1$ per year. As outlined in the Introduction, this sample is still far too small to stringently test theoretical models for the origin of the magnetic field and for the evolution of magnetic white dwarfs.

Unfortunately, discovering new magnetic white dwarfs is a tedious process. Figure 1 shows colour-colour diagrams for all "stellar" objects from the EDR spectroscopic database. It is apparent that the magnetic white dwarfs discussed are well distributed over the locus of hot stars - mainly white dwarfs and subdwarfs. It is, hence, not possible to select magnetic white dwarfs by their colour alone, the availability of a large spectroscopic data set is essential for a significant increase in the number of known magnetic white dwarfs.

In this paper, we have presented fibre spectroscopy of 10 magnetic white dwarf candidates identified in the EDR of the SDSS. We confirm eight of these objects as magnetic DA white dwarfs, of which seven are new discoveries spanning a wide range of magnetic field strengths. This represents a substantial addition to the population of known magnetic white dwarfs. The spectrum of SDSS 2323 contains relatively broad $\mathrm{H} \beta$ and $\mathrm{H} \gamma$ absorption troughs, however, we were not able to confirm the magnetic nature of this star from the SDSS data alone. Finally, we recovered the peculiar magnetic white dwarf HE 0330-0002.

Our eye ball inspection of the SDSS fibre spectra is certainly biased in a number of ways. In white dwarfs with weak magnetic field strengths ( $B \lesssim 1-2 \mathrm{MG}$ ) the resolution and the signal-to-noise $(S / N)$ ratio of the SDSS spectra becomes insufficient to detect the Zeeman splitting of the Balmer lines. Insufficient $S / N$ of the SDSS also lowers the probability of detecting the weak Zeeman absorption components in both hot or high field white dwarfs ( $T_{\text {eff }} \gtrsim 40000 \mathrm{~K}, B \gtrsim 80 \mathrm{MG}$ ). Finally we have restricted or analysis to magnetic white dwarfs with a pure hydrogen atmosphere - and discarded a handful of SDSS spectra with absorption features that could not be identified either with typical stellar transitions or with hydrogen Zeeman components for a wide range of field strengths. We are, however, confident that our selection of magnetic white dwarfs is complete for stars with pure hydrogen atmospheres, magnetic fields of a few MG to a few tens MG, and effective temperatures $10000 \lesssim T_{\text {eff }} \lesssim 40000 \mathrm{~K}$. An a posteriori check of the EDR database using the lists of Jordan (2001) and Wickramasinghe \& Ferrario (2000) confirmed that KUV $03292+0035$ and HE 0330-0002 are the only previously known magnetic white dwarfs with spectroscopic coverage. 
The Early Data Release represents $\sim 5 \%$ of the final SDSS data set. Accounting for our selection bias, we conservatively estimate that the complete SDSS will increase the number of known magnetic white dwarfs by at least a factor 3. Scaling the number of white dwarfs identified by Stoughton et al. (2002 in the EDR sample (734) to the complete SDSS, and assuming $\sim 2-4 \%$ as ratio of magnetic to non-magnetic white dwarfs (Jordan 2001; Wickramasinghe \& Ferrario 2000), suggests that a careful exploitation of the entire SDSS data base, including intense spectroscopic and polarimetric follow-up observations, may well lead to the discovery of several hundred new magnetic white dwarfs.

Acknowledgements. BTG was supported by a PPARC Advanced Fellowship, FE was supported by the DLR under grant 50 OR 99036 , SJ was supported by the DLR under grant 50 OR 0201. We thank the referee, Pierre Bergeron, for suggesting the comparison of the observed SDSS EDR colours with the theoretical white dwarf cooling tracks, and for providing the synthetic $u^{*} g^{*} r^{*} i^{*} z^{*}$ colours used in the bottom panel of Fig. 1. Susanne Friedrich is acknowledged for providing the coordinates of most previously known white dwarfs.

The Sloan Digital Sky Survey (SDSS) is a joint project of the University of Chicago, Fermilab, the Institute for Advanced Study, the Japan Participation Group, the Johns Hopkins University, the Max-Planck-Institut für Astronomie, New Mexico State University, Princeton University, the United States Naval Observatory, and the University of Washington. Apache Point Observatory, site of the SDSS, is operated by the Astrophysical Research Consortium. Funding for the project has been provided by the Alfred P. Sloan Foundation, the SDSS member institutions, the National Aeronautics and Space Administration, the National Science Foundation, the U.S. Department of Energy, Monbusho, and the Max Planck Society. The SDSS World Wide Web site is http://www. sdss.org/.

\section{References}

Achilleos, N., Remillard, R. A., \& Wickramasinghe, D. T. 1991, MNRAS, 253, 522

Bergeron, P., Saffer, R. A., \& Liebert, J. 1992, ApJ, 394, 228

Euchner, F., Jordan, S., Beuermann, K., Gänsicke, B. T., \& Hessmann, F. V. 2002, A\&A, 390, 633

Finley, D. S., Koester, D., \& Basri, G. 1997, ApJ, 488, 375

Fukugita, M., Ichikawa, T., Gunn, J. E., et al. 1996, AJ, 111, 1748

Hagen, H.-J., Groote, D., Engels, D., et al. 1987, A\&A, 183, L7

Harris, H. C., Hansen, B. M. S., Liebert, J., et al. 2001, ApJ, 549, L109

Jordan, S. 1992, A\&A, 265, 570

Jordan, S. 1993, in White Dwarfs: Advances in Observation and Theory, ed. M. A. Barstow, NATO ASIC Proc. No. 403 (Dordrecht: Kluwer), 333

Jordan, S. 2001, in 12th European Workshop on White Dwarfs, ed. J. L. Provencal, H. L. Shipman, J. MacDonal, \& S. Goodchild, ASP Conf. Ser., 226, 269

Margon, B., Anderson, S. F., Harris, H. C., et al. 2002, AJ, 124, 1651

Putney, A. 1995, ApJ, 451, L67

Putney, A. 1997, ApJS, 112, 527

Reimers, D., Jordan, S., Beckmann, V., Christlieb, N., \& Wisotzki, L. 1998, A\&A, 337, L13

Reimers, D., Jordan, S., Koester, D., et al. 1996, A\&A, 311, 572

Reimers, S., Jordan, T., Koehler, L., \& Wisotzki, D. 1994, A\&A, 285, 995

Schmidt, G. D., \& Smith, P. S. 1995, ApJ, 448, 305

Schmidt, G. D., Vennes, S., Wickramasinghe, D. T., \& Ferrario, L. 2001, MNRAS, 328, 203

Stoughton, C., Lupton, R. H., Bernardi, M., et al. 2002, AJ, 123, 485

Szkody, P., Anderson, S. F., Agüeros, M., et al. 2002, AJ, 123, 430

Wegner, G., Boley, F. I., Swanson, S. R., \& McMahan, R. K. 1987, 501

Wickramasinghe, D. T., \& Ferrario, L. 2000, PASP, 112, 873

York, D. G., Adelman, J., Anderson, J. E., et al. 2000, AJ, 120, 1579 